\title{
Applying Circadian Rhythm Concepts in Digital Healthcare
}

\author{
Chul-Hyun Cho ${ }^{1,2,3}$ and Heon-Jeong Lee ${ }^{3,4}$ \\ 'Department of Psychiatry, College of Medicine, Chungnam National University, Daejeon, Korea \\ 2Department of Psychiatry, Chungnam National University Sejong Hospital, Sejong, Korea \\ ${ }^{3}$ Chronobiology Institute, Korean University, Seoul, Korea \\ ${ }^{4}$ Department of Psychiatry, Anam Hospital, Korea University College of Medicine, Seoul, Korea
}

The era of digital healthcare has arrived; with the development of sensing technology, it has become possible to acquire everyday data through digital phenotyping. Mobile devices have various sensing features, and artificial intelligence (AI)-related technologies that can process and analyze complex [1]. The gap between the technology development and its application in medical practice is narrowing, and research and industrialization related to digital healthcare is exploding.

The actual application of digital healthcare, depends on various criteria such as the clinical departments, diseases, functions, age, and sex. The clinical application of digital healthcare is ultimately determined according to clinical needs. However, it is easy to fail without proper application. Based on the evidence, treatment methods should be digitalized to improve current treatment methods or improve prognosis [2].

Another important aspect is establishing the uniqueness and versatility of the application of digital healthcare technology. This could be an advanced technology or an application based on the understanding of basic human physiology. As an example, an approach based on the circadian rhythm can be considered. Disturbance of the circadian rhythm not only causes sleep disorders, it also has a long and profound effect on the cardiovascular and endocrine systems, which can lead to cancer, degenerative brain disease, and mental diseases [3]. Therefore, the application of digital healthcare based on circadian rhythm is medically sound as well

Received: March 3, 2021 Accepted: March 7, 2021

Corresponding author: Heon-Jeong Lee, MD, PhD, Department of Psychiatry, Anam Hospital, Korea University College of Medicine, 73 Goryeodae-ro, Seongbuk-gu, Seoul 02841, Korea.

Tel: 82-2-920-6721, Fax: 82-2-927-9024, E-mail: leehjeong@korea.ac.kr

(a) This is an Open Access article distributed under the terms of the Creative Commons Attribution Non-Commercial License (https://creativecommons.org/ licenses/by-nc/4.0) which permits unrestricted non-commercial use, distribution, and reproduction in any medium, provided the original work is properly cited. as unique and versatile.

\section{DIAGNOSTIC APPLICATION}

Diagnosis can be divided into a fragmentary diagnosis that measures the current state of the circadian rhythm and a complicated diagnosis that predicts any disease-related form based on circadian rhythm measurements which can be carried out using core body temperature, gene expression, hormone secretion, and actigraphy. However, there were many difficulties in applying such a method to real life. It is now possible to acquire multiple types of information, such as physical activity, sleep, and heart rate, using sensors in smartphones and wearable devices [4]. In the future, with the development of sensing technology, the amount, quality, and types of data that can be acquired easily in daily life will increase. Thus, it will be possible to measure and diagnose various aspects of the circadian rhythm.

Based on this relatively easy method of measuring circadian rhythm-related data, a complicated diagnostic step for classifying or predicting disease-related conditions can be considered. Practical application is possible for health conditions, such as insomnia and diabetes, that are closely related to the circadian rhythm. Attempts for the predictive diagnosis of mental or physical conditions or diseases based on this circadian rhythm are being made gradually, and meaningful results are being derived. In AI analysis methods such as machine learning, it is crucial to classify and organize meaningful data for learning from complex clinical data and have miscellaneous information [5]. In this regard, reclassifying and reprocessing clinical data from the perspective of circadian rhythm will be very useful making it applicable in more diverse areas of medicine. 


\section{THERAPEUTIC APPLICATION}

First, we can think of a digital therapeutic intervention for stabilizing the circadian rhythm through the realignment of the sleep-wake rhythm. This may prioritize therapeutic applications in groups vulnerable to disturbances in sleep-wake rhythms with continuing therapeutic intervention in real life and customized interventions according to individual circadian rhythm states [6]. It is highly likely to be established as a non-pharmacological treatment method and will apply not only to diseases such as insomnia, but also to medical conditions such as diabetes.

The therapeutic application of the circadian rhythm, along with technologies such as the Internet of Medical Things, will radically change human life in terms of treatment and prevention. Beyond fragmentary information such as activity and sleep currently obtained through smart devices, it will provide information on what kind of circadian rhythm the user is in and how much it can affect their health status; inducing integrated interventions in everyday environments can be an important step forward.

The therapeutic application of circadian rhythm measurements in digital health is still in its infancy. Therefore, as diagnostic applications and related data analysis technology develop, therapeutic applications can be expected to advance explosively. Since individual therapeutic applications based on unique circadian rhythm patterns are possible, it will be a sound basis for personalized treatment, which is one of the critical characteristics of future medicine [7].

\section{APPLICABILITY ON HEALTHCARE SYSTEM}

Hospitals and other nursing facilities may be representative of healthcare facilities. Hospitals could be modified to accommodate the study of circadian rhythm in a specific facility or department.

First, we consider the settings in the intensive care unit (ICU), where patients with relatively severe diseases are admitted to receive intensive therapeutic care, so problems such as delirium frequently occur [8]. In the ICU, it is either difficult to obtain enough light during the day or challenging to construct a complete lightblocking environment at night. Therefore, the circadian rhythm can be easily disturbed. Using digital healthcare technology, improvements can be made to create a healthy environment based on the circadian rhythm.

Second, we can think of the psychiatric closed ward hospitalization environment [9]. A significant number of mental disorders are closely related to circadian rhythm disturbances. Patients with mental illness often have sleep-wake problems. However, similar to the ICU, the closed ward must maintain a closed space for the patient's treatment and security. Exposure to light or controlling the amount of activity to maintain a stable circadian rhythm may be problematic due to these environmental constraints. It is possible to measure each patient's circadian rhythm state in the closed ward, predict the clinical condition, and apply chronother- apeutic interventions for circadian rhythm stabilization.

Next, we consider the settings for shift workers [10]. Hospitals have essential medical personnel who have to work shifts, which can cause significant illnesses and increase the risk of medical accidents. Creating a more effective and safer shift work environment is essential for improving the quality of medical care, protecting personnel's health, and preventing accidents. Digital technology can be fully applied to patients and medical personnel who treat patients directly or indirectly.

\section{CONCLUSION}

The greater the volume and versatility of data, the more crucial it is to extract and utilize clinically meaningful information from it. Additionally, deciding where and how to apply digital technology determines the success or failure of clinical applications. The circadian rhythm can provide us with a valuable perspective for the use of data in clinical applications. Thus, it is expected that more useful and practical digital healthcare applications will be possible.

\section{Acknowledgments}

This work was supported by a National Research Foundation of Korea (NRF) grant funded by the Government of Korea (MSIT) (NRF-2020R1C1C1007463), and the Brain Research Program through the National Research Foundation of Korea (NRF) funded by the Ministry of Science, ICT \& Future Planning (NRF2016M3C7A1914448).

\section{Conflicts of Interest}

The authors have no potential conflicts of interest to disclose.

\section{ORCID iDs}

Chul-Hyun Cho (1)

https://orcid.org/0000-0002-1663-9680

Heon-Jeong Lee (1)

https://orcid.org/0000-0002-9560-2383

\section{REFERENCES}

1. Cho $\mathrm{CH}$, Lee HJ. Could digital therapeutics be a game changer in psychiatry? Psychiatry Investig 2019;16:97-98.

2. Graham AK, Lattie EG, Mohr DC. Experimental therapeutics for digital mental health. JAMA Psychiatry 2019;76:1223-1224.

3. Walker WH 2nd, Walton JC, DeVries AC, Nelson RJ. Circadian rhythm disruption and mental health. Transl Psychiatry 2020;10:28.

4. Lee HA, Lee HJ, Moon JH, Lee T, Kim MG, In H, et al. Comparison of wearable activity tracker with actigraphy for sleep evaluation and circadian restactivity rhythm measurement in healthy young adults. Psychiatry Investig 2017;14:179-185.

5. Topol EJ. High-performance medicine: the convergence of human and artificial intelligence. Nat Med 2019;25:44-56.

6. Cho CH, Lee T, Lee JB, Seo JY, Jee HJ, Son S, et al. Effectiveness of a smartphone app with a wearable activity tracker in preventing the recurrence of mood disorders: prospective case-control study. JMIR Ment Health 2020;7: e21283.

7. Agostinelli F, Ceglia N, Shahbaba B, Sassone-Corsi P, Baldi P. What time is 
it? Deep learning approaches for circadian rhythms. Bioinformatics 2016; 32:i8-i17.

8. Engwall M, Fridh I, Johansson L, Bergbom I, Lindahl B. Lighting, sleep and circadian rhythm: an intervention study in the intensive care unit. Intensive Crit Care Nurs 2015;31:325-335.
9. Loving RT, Kripke DF. Daily light exposure among psychiatric inpatients. J Psychosoc Nurs Ment Health Serv 1992;30:15-19.

10. Monk TH. What can the chronobiologist do to help the shift worker? J Biol Rhythms 2000;15:86-94. 